\title{
X-ray Absorption Spectroscopy with other simulation techniques to work on amorphous and nano-systems
}

\author{
Author: Yuanpeng Zhang ${ }^{1,2}$ \\ ${ }^{1}$ School of Physics and Astronomy, Queen Mary, University of London, London, UK E1 4NS \\ ${ }^{2}$ Spallation Neutron Source, Oak Ridge National Laboratory, Oak Ridge, TN 37830
}

X-ray absorption spectroscopy (XAS) is a local environment sensitive technique, therefore, it can be used to explore the structure of systems lacking long range order. Here XAS was used together with the density functional theory (DFT) calculation and molecular dynamics (MD) simulations to comprehensively study amorphous and nano-systems. Firstly, the extended X-ray absorption fine structure (EXAFS) was combined with DFT calculation and several other experimental techniques, including optical transmission microscopy and Raman spectroscopy, to reveal the phase transition behavior of Ge quantum dots (QDs, $\sim 4 \mathrm{~nm}$ ) under hydrostatic high pressure. It was shown that the Ge QDs undergoes a low-density-amorphous to high-density-amorphous type of phase transition. Secondly, the theoretical EXAFS calculation was combined with the MD simulation to work on both the amorphous GaSb (aGaSb) and Ge QDs systems. To realize the automatic flow from the MD to EXAFS simulation, a program called MD2FEFF was developed. Here aGaSb was used as a reference system, and the results demonstrated the validity of both the combined MD+EXAFS simulation approach and the activation-relaxation technique to generate the aGaSb model. For the Ge QDs, the combined simulation approach reveals that our annealed Ge QDs could be best described by a core-shell model where the core is crystalline and shell is amorphous. 